\title{
Controlled Functionalisation of 2-D Materials for Quantum Device Development: assessment of Single Atom Behaviour via Atomic Resolution Electron Microscopy and Spectroscopy
}

Ursel Bangert ${ }^{1}$, Michael Hennessy ${ }^{1}$, Eoghan O'Connell $^{1}$, Eileen Courtney ${ }^{1}$, Eoin Moynihan ${ }^{1}$, Alan Harvey $^{1}$, Quentin Ramasse ${ }^{2}$, Demie Kepaptsoglou ${ }^{2}$, Hans Hofsaess ${ }^{3}$, Manuel Auge ${ }^{3}$, Stefan Rost ${ }^{4}$ and Beata Kardynal $^{4}$

${ }^{1}$ University of Limerick, Limerick, Limerick, Ireland, ${ }^{2}$ SuperSTEM Laboratory and University of Leeds, Daresbury, England, United Kingdom, ${ }^{3}$ Georg-August-Universität, Göttingen, Niedersachsen, Germany, ${ }^{4}$ Forschungszentrum Juelich, Juelich, Nordrhein-Westfalen, Germany

Controlled functionalisation of nano- and low-dimensional materials will open possibilities for fabrication of innovative devices, e.g., long aspired single photon sources and devices for quantum metrology based on these materials. Achievement of these is very likely, if nanoscale localised electronic doping compatible with large-scale integrated semiconductor technologies can be achieved. Targeted doping, of nano-tubes and 2-D materials, e.g., graphene and monolayer $\mathrm{BN}$ as well as transition metal dichalcogenides (TMDs), has been attempted here via ultra-low energy ion implantation in order to tailor bandstructure and thus opto-electronic properties [1, 2, 3, 4]. TMDs are particularly promising for quantum device purposes, as they possess many unique properties, such as large direct bandgaps, optically addressable spin and valley pseudospin degrees of freedom and large magnetic moments.

By using atomic resolution high angle annular dark field (HAADF) scanning transmission electron microscopy (STEM) supported by structure modelling and image simulation, as well as atomic-scale electron energy loss spectroscopy (EELS), we show that precise sites (e.g., substitutional, interstitial, adatom, vacancy), nature and dynamics (e.g., mobility) of individual implants/dopants/point defects in 2-Ds can uniquely and directly be identified. Custom software, based on Atomap [5], is employed to automatically identify atomic sites in experimental HAADF STEM images. Atomic positions and intensities are used, to create accurate image simulations. Agreement between experimental and simulated images then provides proof of individual implant positions. Ab initio band structure calculations are furthermore used to analyse the suitability of 2-Ds, especially TMDCs, for electronic tailoring via ion implantation. Evidence of highly localized bandstructure modification as well as of plasmon tailoring due to foreign atoms in graphene and TMDCs will be presented. The examples shown of implants into TMDCs, e.g., Se and $\mathrm{Cr}$ in $\mathrm{MoSe}_{2}, \mathrm{WS}_{2}$ and $\mathrm{WSe}_{2}$, are part of a broad study into the creation of single photon emitters in TMDs. Low loss EELS, as well as Photoluminescence and Raman spectroscopy were employed to study exciton behavior in these implanted TMDs, supported by Hanbury Brown and Twiss experiments to verify single photon emission from implantation sites [6].

\section{References}

[1] U. Bangert, W. Pierce, D. M. Kepaptsoglou, Q. Ramasse, R. Zan, M. H. Gass, J. A. Van den Berg, C. B. Boothroyd, J. Amani, H. Hofsäss, , Nano Lett. 13 (10), 4902 (2013)

[2] U. Bangert, W. Pierce, C. Boothroyd, C.-T. Pan, R. Gwilliam, Nature Scientific Reports www.nature.com/articles/srep27090

[3] D. Kepaptsoglou, T. Hardcastle, C. Seabourne, U. Bangert, R. Zan, J. Amani, H. Hofsäss, R. Nicholls, R. Brydson, A. Scott, Q. Ramasse, ACSNano9 (11), 11398-11407 (2015) 
[4] U. Bangert, A. Stewart, E. O’Connell, E. Courtney, Q. Ramasse, D. Kepaptsoglou, H. Hofsäss, J. Amani, S.-S. Tu, B. Kardynal, Ultramicroscopy, 176, 31-36 (2017)

[5]. M. Nord, P. E. Vullum, I. MacLaren, T. Tybell, and R. Holmestad, Adv. Struct. Chem. Imaging 3, 9 (2017).

[6]. P. Tonndorf, R. Schmidt, R. Schneider, J. Kern, M. Buscema, G. A. Steele, A. Castellanos-Gomez, H. S. J. van der Zant, S. Michaelis de Vasconcellos, and R. Bratschitsch, Optica 2, 347 (2015). 